\title{
Yeast as a model of human mitochondrial tRNA base substitutions: Investigation of the molecular basis of respiratory defects
}

\author{
ARIANNA MONTANARI, ${ }^{1,6}$ CÉLINE BESAGNI, ${ }^{2,6}$ CRISTINA DE LUCA, ${ }^{1}$ VERONICA MOREA, ${ }^{3}$ ROMINA OLIVA,

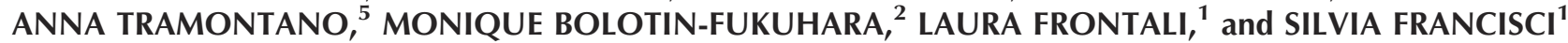 \\ ${ }^{1}$ Department of Cell and Developmental Biology, Pasteur Institute-Fondazione Cenci Bolognetti, University Sapienza, 00185 Rome, Italy \\ ${ }^{2}$ Laboratoire de Génétique Moléculaire, Institut de Genétique et Microbiologie UMR CNRS 8621, Université Paris Sud, 91405 Orsay Cedex, France \\ ${ }^{3}$ CNR-Institute of Molecular Biology and Pathology (IBPM), Pasteur Institute-Fondazione Cenci Bolognetti, University Sapienza, 00185 Rome, Italy \\ ${ }^{4}$ Department of Applied Sciences, University Parthenope, 80133 Naples, Italy \\ ${ }^{5}$ Department of Biochemical Sciences, Pasteur Institute-Fondazione Cenci Bolognetti, University Sapienza, 00185 Rome, Italy
}

\begin{abstract}
We investigate the relationships between acylation defects and structure alterations due to base substitutions in yeast mitochondrial (mt) tRNA UUR. The studied substitutions are equivalent to the A3243G and T3250C human pathogenetic tRNA mutations. Our data show that both mutations can produce tRNA Leu acylation defects, although to a different extent. For mutant A14G (equivalent to MELAS A3243G base substitution), the presence of the tRNA and its defective aminoacylation could be observed only in the nuclear context of W303, a strain where the protein synthesis defects caused by tRNA base substitutions are far less severe than in previously studied strains. For mutant T20C (equivalent to the MM/CPEO human T3250C mutation), the acylation defect was less severe, and a thermosensitive acylation could be detected also in the MCC123 strain. The correlation between the severity of the in vivo phenotypes of yeast tRNA mutants and those obtained in in vitro studies of human tRNA mutants supports the view that yeast is a suitable model to study the cellular and molecular effects of tRNA mutations involved in human pathologies. Furthermore, the yeast model offers the possibility of modulating the severity of yeast respiratory phenotypes by studying the tRNA mutants in different nuclear contexts. The nucleotides at positions 14 and 20 are both highly conserved in yeast and human mt tRNAs; however, the different effect of their mutations can be explained by structure analyses and quantum mechanics calculations that can shed light on the molecular mechanisms responsible for the experimentally determined defects of the mutants.
\end{abstract}

Keywords: Saccharomyces cerevisiae; mitochondria; tRNA acylation and structure; human mitochondrial pathologies; yeast model

\section{INTRODUCTION}

Human mitochondrial (mt) tRNA genes are frequently affected by pathogenic mutations, mainly substitutions, but also base deletions and duplications. However, the molec-

\footnotetext{
${ }^{6}$ These authors contributed equally to this work.

Abbreviations: $\mathrm{mt}$, mitochondrial; cyt, cytoplasmic; MELAS, mitochondrial encephalomyophathy, lactic acidosis, and stroke-like episodes; MM/ $\mathrm{CPEO}$, mitochondrial myopathy/chronic progressive external ophthalmoplegia; TUF1, gene encoding for the mitochondrial protein synthesis elongation factor (EF-Tu); RH, reverse Hoogsten; syn-, mitochondrial mutation that leads to mitochondrial protein synthesis deficiency; rho, indicates the state of the mitochondrial DNA: rho ${ }^{+}$(wild-type), rho ${ }^{\circ}$ (absent), rho- (large DNA deletion).

Reprint requests to: Silvia Francisci, Department of Cell and Developmental Biology, University of Rome I, Piazzale A. Moro 5, 00185 Rome, Italy; e-mail: silvia.francisci@uniroma1.it; fax: +39 064461980.

Article published online ahead of print. Article and publication date are at http://www.rnajournal.org/cgi/doi/10.1261/rna.740108.
}

ular consequences on tRNA structure and function are still largely unclear, and so are the molecular mechanisms at the basis of the defective phenotypes resulting from these mutations. In vivo studies are complicated by a varying degree of heteroplasmy and variable nuclear genetic background, as well as limited by the availability of biopsy material. We have previously suggested that the molecular and cellular effects of the mt "pathological" tRNA mutations can be effectively studied in yeast (Feuermann et al. 2003). Mt function is actually not necessary in Saccharomyces cerevisiae, and very severe mt defects can be studied in viable fermenting cells.

Base substitutions can be inserted into yeast $\mathrm{mt}$ tRNA genes by biolistic procedures, and we have previously shown that the three yeast $m t$ tRNA $\mathrm{LuU}$ substitutions, A14G, C26T, and T69(60)C, equivalent to MELAS 
disease-associated mutations (A3243G, C3256T, and T3291C in human, respectively) specifically produce very severe respiratory defects. The number reported in parentheses refers to the standard sequence numbering (yeast cytoplasmic [cyt] tRNA ${ }^{\text {Phe }}$ [76 nucleotides] generally used as a reference for tRNA molecules).

The respiratory phenotype of the above yeast mutants was accompanied by rapid loss of mtDNA, as may be expected by a strong inhibition of $\mathrm{mt}$ protein synthesis (Myers et al. 1985). The studied substitutions caused respiratory phenotypes with different degrees of severity, all of which were suppressed by overexpression of the $\mathrm{mt}$ protein synthesis elongation factor EF-Tu and of the $\mathrm{mt}$ leucyl-tRNA synthetase. Due to the rapid loss of mtDNA in these mutants, Northern experiments could reveal the presence of the mutated tRNA $\mathrm{UUR}_{\mathrm{UUR}}^{\mathrm{Leu}}$ only in cells transformed with plasmids bearing the above suppressor genes (Feuermann et al. 2003; De Luca et al. 2006). Since this experimental system is not ideal to study the molecular mechanisms causing the defective phenotypes, we decided to investigate these mechanisms in conditions where the protein synthesis is less severely affected and the presence of suppressors is not necessary for the maintenance of the mutated mtDNA. To this end, we exploited our observation that the same mutation could produce different effects in yeast cells with different nuclear contexts (De Luca et al. 2006).

In the present work, we explore the possibility of using yeast as a model system to study the molecular mechanisms causing respiratory defects of human equivalent tRNA mutations, and we focus our attention on two yeast $\mathrm{mt}$ tRNA ${ }_{\text {UUR }}^{\text {Leu }}$ base substitutions, A14G and T20C, equivalent to $\mathrm{A} 3243 \mathrm{G}$ and $\mathrm{T} 3250 \mathrm{C}$ human tRNA $\mathrm{LuUR}$ pathogenetic mutations. The structure of mitochondrial tRNA $\mathrm{LUU}_{\mathrm{UUR}}^{\mathrm{Leu}}$ is very similar in human and yeast mitochondria except for the presence of a much longer variable loop in yeast tRNA. However, analysis of the available structural data shows that this difference should not influence the interactions of nucleotides 14 and 20 . The severity of the phenotypes of the A14G and T20C yeast mutants parallels that reported for the equivalent human tRNA $A_{U U R}^{\text {Leu }}$ pathogenetic mutations. We also find that the percentage of rho $^{\circ}$ colonies is a very good indicator of the severity of the respiratory phenotype, and that this in turn can vary to a significant extent in different yeast strains. This prompted us to study the effects of the A14G mutation, causing a particularly severe phenotype, in a different nuclear context where its effects are less severe. We study the in vivo aminoacylation of tRNAs by using partially denaturing polyacrylamide-urea acidic sequencing gels, which were previously proven to be a suitable tool to provide information on the acylation state of tRNA molecules (Francisci et al. 2005). Finally, an analysis of yeast and human tRNA sequences and structures associated with quantum mechanics calculations allows us to derive some insights into the structural role of the A14 and T20 nucleotides of yeast and human tRNA $\mathrm{Lu}$.eu .

\section{RESULTS}

The $m t$ tRNA UUU Lequence is very similar in human and $S$. cerevisiae. Figure 1 shows the comparison of the cloverleaf structures of the two tRNAs and the mutations introduced in yeast tRNA $A_{U U R}^{\mathrm{Leu}}$ by a biolistic procedure. The substitutions studied herein, in positions 14 and 20, are marked with circles; MELAS base substitutions are underlined.

\section{Acylation defects can be detected by Northern analysis}

During the characterization of human equivalent base substitutions, we observed a decrease of respiration and a delayed growth on glycerol-containing medium of the yeast strain MCC123 carrying the T20C base substitution in the mt tRNA Leu (M/T20C) (Table 1; Besagni 2007). This mutation is located in the $\mathrm{D}$ loop, at position 20 (numbering follows that proposed by Sohm et al. 2003) and is equivalent to the human pathogenic mutation T3250C, present in a family with mt myopathy (MM/CPEO) (Goto et al. 1992).

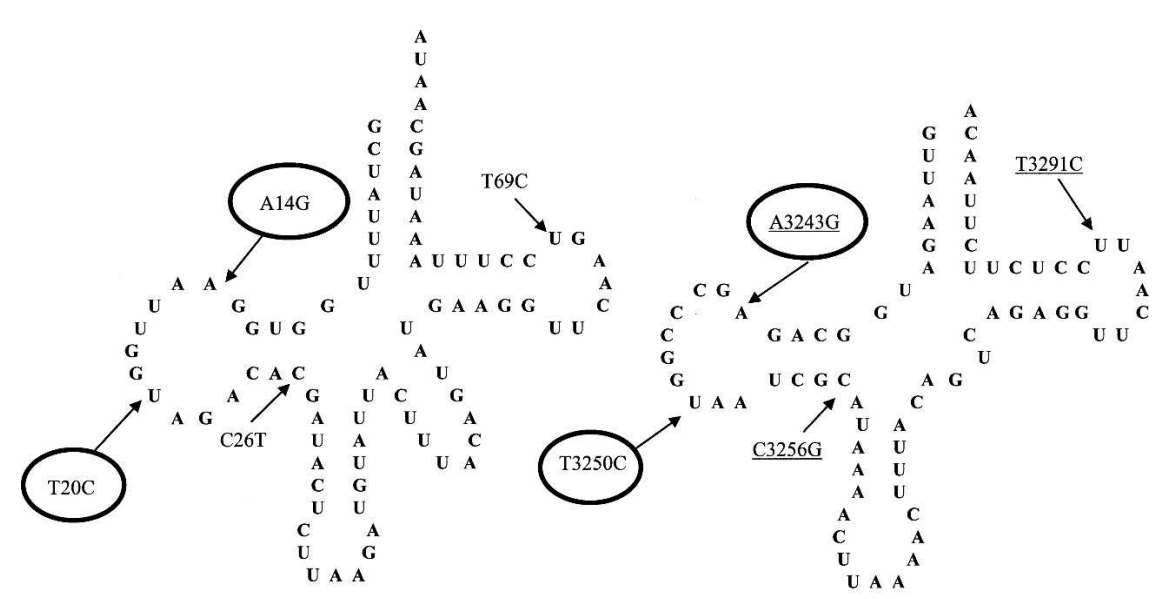

S. cerevisiae Homo sapiens

FIGURE 1. Comparison of yeast and human mt tRNA Leu cloverleaf structures and mutations introduced in yeast mt tRNA Leu by biolistic procedures. (Arrows) The "pathological mutations" described in Feuermann et al. (2003) and equivalent to MELAS base substitution (underlined) and the mutations analyzed in this work (circles). 
TABLE 1. S. cerevisiae strains used in this study

\begin{tabular}{|c|c|c|}
\hline Strain nuclear genotype & Mitochondrial genotype & Reference \\
\hline MCC123 (Mat a, ade2-1, ura3-52, kar1-1) & $\mathrm{Rho}^{+}$and $\mathrm{Rho}^{\circ}$ & Mulero and Fox (1993) \\
\hline M/A14G id & Syn- (tRNA $\left.{ }^{\text {Leu }} \mathrm{A} 14 \mathrm{G}\right)$ & This paper \\
\hline M/T20C id & Syn- (tRNA ${ }^{\text {Leu }}$ T20C) & Besagni (2007); this paper \\
\hline $\begin{array}{l}\text { W303-1B (Mat } \alpha \text {, his3-11, ade2-1, leu2-3,112, } \\
\text { ura3-1, trp1- } \Delta 2 \text {, can1-100) }\end{array}$ & $\mathrm{Rho}^{+}$and $\mathrm{Rho}^{\circ}$ & $\begin{array}{l}\text { Thomas and Rothsein (1989); } \\
\text { this paper }\end{array}$ \\
\hline W/A14G id & Syn- (tRNA $\left.{ }^{\text {Leu }} \mathrm{A} 14 \mathrm{G}\right)$ & This paper \\
\hline W/T20C id & Syn- (tRNA ${ }^{\text {Leu }}$ T20C) & This paper \\
\hline D273-10B/A1 (Mat $\alpha$, met6) & $\mathrm{Rho}^{+}$ & Berlani et al. (1980) \\
\hline $\mathrm{D} / \mathrm{A} 14 \mathrm{G}$ id & Syn- (tRNA ${ }^{\text {Leu }}$ A14G) & This paper \\
\hline $\mathrm{D} / \mathrm{T} 20 \mathrm{C}$ id & Syn - (tRNA ${ }^{\text {Leu }}$ T20C) & This paper \\
\hline
\end{tabular}

Examination of mt tRNA by Northern analysis showed that the M/T20C mutant produces a normal amount of tRNA LuU compared with the wild type, but that tRNA exhibits a faster electrophoretic migration (Fig. 2, top panel), indicating that a conformational change in the three-dimensional structure had occurred. Additionally, in the mitochondria from wild-type and mutant cells grown at $28^{\circ} \mathrm{C}$, the deacylated molecule of $\mathrm{tRNA}^{\mathrm{Leu}}$, migrating faster, could be distinguished from the aminoacylated form. However, the deacylation treatment, shown in the $\mathrm{D}$ lane, had no effect on the mutated tRNA ${ }^{\text {Leu }}$ extracted from $\mathrm{M} / \mathrm{T} 20 \mathrm{C}$ cells grown at $37^{\circ} \mathrm{C}$, indicating that they have an aminoacylation defect. This defect is specific for tRNA $\mathrm{UUR}_{\mathrm{UU}}^{\mathrm{Leu}}$, since mt RNA samples hybridized with the VAL probe, as a control, did not show this defect either at $28^{\circ} \mathrm{C}$ or $37^{\circ} \mathrm{C}$ (Fig. 2, bottom panel).

From these results, we can conclude that the M/T20C mutant has a thermosensitive aminoacylation, which parallels the moderate severity of the aminoacylation defect obtained for the human $\mathrm{T} 3250 \mathrm{C}$ variant in an in vitro acylation assay, compared with wild type and the human A3243 variant. The latter result has been interpreted by postulating that T3250 plays the role of a minor identity element (Sohm et al. 2003).

\section{Base substitutions result in different phenotypes in different nuclear contexts}

We have previously reported that the nuclear background can have a significant effect on the phenotype of mt tRNA mutants (De Luca et al. 2006). In this work, we explore the possibility of identifying the molecular defect caused by tRNA $^{\text {Leu }}$ mutations associated with human pathologies that could not be identified in previous studies due to the rapid loss of mtDNA (Feuermann et al. 2003).

By a cytoductant cross, we transferred the mitochondria carrying the A14G mutation from the originally studied severely respiratory deficient strain to several others and used the $\mathrm{rho}^{\circ}$ production as an indicator of the importance of the mt protein synthesis defect. Results reported in Table 2 show that the cells with mitochondria harboring the tRNA Leu A14G mutation are unable to grow in glycerolcontaining media in the W303 nuclear context, as they did in the original MCC123 strain, but originated a far lower number of $\mathrm{rho}^{\circ}$ colonies (10\% compared with $\left.80 \%\right)$. Figure 3 reports the Northern analysis of tRNA $\mathrm{LuU}_{\mathrm{UU}}^{\mathrm{Lu}}$, performed with RNAs extracted from purified mitochondria of the W303-1B wild-type and W/A14G strains and hybridized with the LEU probe. The mutated tRNA $\mathrm{LUU}_{\mathrm{UU}}^{\mathrm{Lu}}$ was detectable after overexposure of the right part of the filter containing the W/A14G samples. The majority of the tRNA $\mathrm{LUU}_{\mathrm{UUR}}$ was in the deacylated form, but a slower aminoacylated fraction was visible (Fig. 3, indicated by an arrow). The faint hybridization observed after long exposure was obtained with a very specific probe, and the absence of
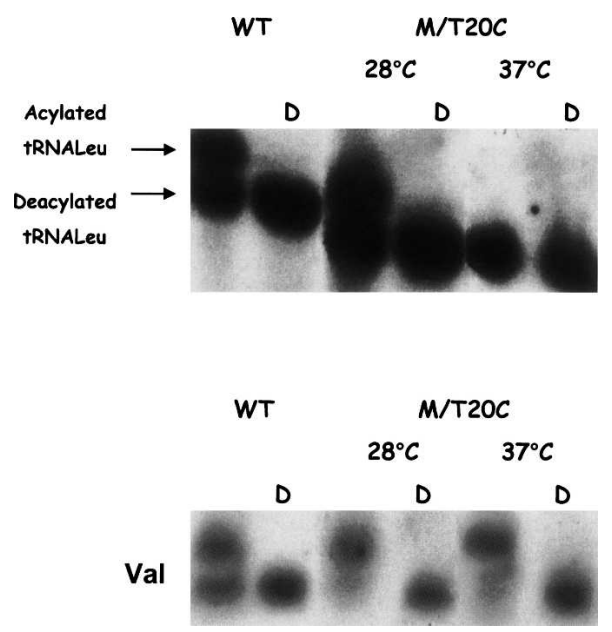

FIGURE 2. Northern blot analysis of mt transcripts. Total RNAs were extracted under acidic conditions from purified mitochondria isolated from the indicated mutants or wild-type strains and fractionated on polyacrylamide-urea partially denaturing gels. (D lanes) Samples treated to deacylate the tRNAs. Hybridization was with the 5 '-end-labeled LEU-probe (top panel) and, as control, 5' -end-labeled VAL probe (bottom panel). RNAs were extracted from MCC123 and $\mathrm{M} / \mathrm{T} 20 \mathrm{C}$ mutant cells grown at $28^{\circ} \mathrm{C}$ and $37^{\circ} \mathrm{C}$. 
TABLE 2. Characteristics of studied strains

\begin{tabular}{|c|c|c|c|c|}
\hline Nuclear context & $\begin{array}{c}\text { Base } \\
\text { substitution }\end{array}$ & $\begin{array}{l}\mathrm{Rho}^{-} / \text {Rho }^{\circ} \\
\text { percentage }\end{array}$ & $\begin{array}{l}\text { Glycerol growth } \\
\text { at } 28^{\circ} \mathrm{C}\end{array}$ & tRNA ${ }^{\text {Leu }}$ Northern analysis \\
\hline MCC123 & - & $2 \%$ & ++ & No defect \\
\hline MCC123 & A14G & $80 \%$ & - & No tRNAs \\
\hline MCC123 & A14GpTUF1 ${ }^{a}$ & $5 \%$ & ++ & No defective aminoacylation \\
\hline MCC123 & T20C & $35 \%$ & $+/-$ & Thermosensitive aminoacylation \\
\hline W303-1B & - & $2 \%$ & +++ & No defect \\
\hline W303-1B & A14G & $10 \%$ & - & Severe aminoacylation defect already at $28^{\circ} \mathrm{C}$ \\
\hline W303-1B & T20C & $13 \%$ & ++ & $\begin{array}{l}\text { Low amount of aminoacylated form both at } 28^{\circ} \mathrm{C} \\
\text { and } 37^{\circ} \mathrm{C}\end{array}$ \\
\hline D273-10B/A1 & - & $2 \%$ & +++ & Not determined \\
\hline $\mathrm{D} 273-10 \mathrm{~B} / \mathrm{A} 1$ & A14G & $8.5 \%$ & - & Not determined \\
\hline $\mathrm{D} 273-10 \mathrm{~B} / \mathrm{A} 1$ & T20C & $2 \%$ & - & Not determined \\
\hline
\end{tabular}

All mutated tRNA ${ }^{\text {Leu }}$ exhibit a change in electrophoretic migration, indicating a structural modification of the molecules.

aPresence of multicopy plasmid bearing the TUF1 suppressor gene.

cytoplasmic contamination was verified (see Materials and Methods).

Moreover, the different electrophoretic migration of wild-type and mutated tRNA ${ }^{\text {Leu }}$ indicated a conformational change of the molecule. This result is significantly different from what we observed in M/A14G strain. In this case, the $\mathrm{mt}$ tRNAs were undetectable.

We observed a similar effect when the mitochondria bearing the T20C mutation, mentioned above, were transferred from the MCC123 to the W303-1B nuclear context by a similar cytoduction procedure. Also, in this case, we observed a relevant decrease of $\mathrm{rho}^{\circ}$ production with respect to the original MCC123 context (Table 2).

\section{Sequence and structure analysis of yeast tRNA bearing the $A 14 G$ or the $\mathrm{T} 20 \mathrm{C}$ mutation}

Positions 14 and 20 of tRNA Leu are both located in the D loop; therefore, they are most likely not involved in basepair interactions necessary for the maintenance of the secondary structure of the tRNA.

The most relevant difference between the human and $S$. cerevisiae $\mathrm{mt}$ tRNA $\mathrm{LuU}$ sequences reported in Figure 1 is the length of the $\mathrm{V}$ loop, based on which the yeast and human mt tRNA $\mathrm{LUU}_{\mathrm{UU}}^{\mathrm{Lu}}$ are assigned to class II and I tRNAs, respectively. Since nucleotides occurring at positions 14 and 20 or nucleotides involved in conserved interactions with them are not involved in interactions with the $\mathrm{V}$ loop in known tRNA structures, the different length of the $\mathrm{V}$ loop in yeast and human tRNA $\mathrm{UUU}_{\mathrm{UU}}^{\mathrm{Lu}}$ is not expected to affect the interactions involving these nucleotides. Consequently, the effect of the A14G and T20C mutations on yeast and human tRNA $\mathrm{LUR}_{\mathrm{UUR}}^{\mathrm{Leu}}$ is expected to be essentially the same.

A14 is one of the 15 invariant nucleotides of "canonical" tRNAs (Bloomfield et al. 2000) and, in known tRNA structures, is involved in a conserved reverse Hoogsten ( $\mathrm{RH})$ base pair with the invariant nucleotide U8 (Fig. 4). In some class I tRNAs, such as yeast tRNA ${ }^{\text {Asp }}, \mathrm{A} 14$ also forms hydrogen bonds with A21 to give a A14-U8-A21 base triplet. This tertiary interaction might also be present in human, but not in yeast, tRNA $\mathrm{Leu}$.

The A14-U8 RH interaction does not belong to either of the tRNA secondary structure motifs (the double-helix AA stem-T arm or AC arm-D arm). It is a tertiary interaction that seems to play a key role in stabilizing the $D$ arm internal loop connecting the two secondary structure motifs, and therefore in maintaining these motifs in the correct relative orientation with respect to each other (Fig. 4A,B). Quantum mechanics (QM) energy calculations were performed on the A14-U8 base pair starting from geometry they have in the crystal structure of Escherichia coli tRNA $^{\text {Phe }}$ (PDB ID: 1EHZ), and on the G14-U8 base pair modeled by replacing the adenine in position 14 with a

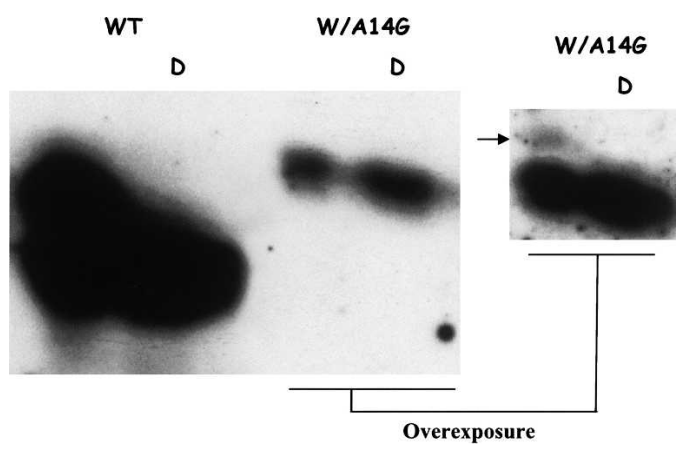

FIGURE 3. Northern blot analysis of $\mathrm{mt}$ transcripts. Total RNAs were extracted under acidic conditions from purified mitochondria isolated from W303-1B (WT) or mutant W/A14G strains grown in galactose at $28^{\circ} \mathrm{C}$. Mt tRNAs were fractionated on polyacrylamideurea partially denaturing gels. (D lanes) Samples treated to deacylate the tRNAs. Hybridization was with the $5^{\prime}$-end-labeled LEU probe. The overexposed autoradiograph, containing the W/A14G samples, is shown at the right. (Arrow) The acylated tRNA $A_{U U R}^{\mathrm{Leu}}$. 
A

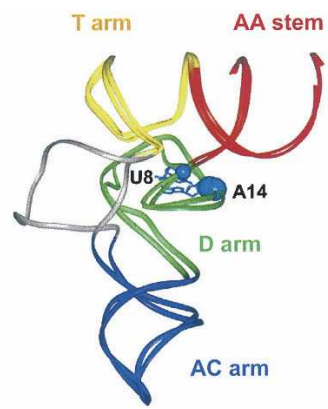

C

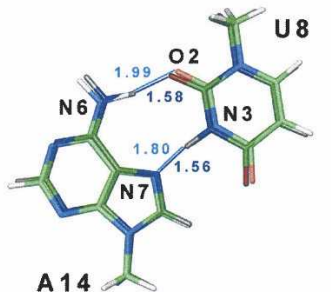

B

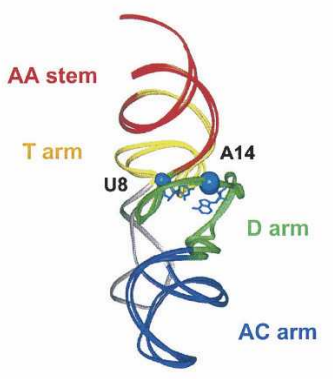

D

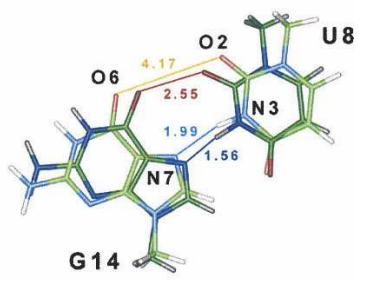

FIGURE 4. Structural effect of the A14G mutation. $(A, B)$ A14-U8 base pair mapped on the superimposed 3-D structures of representative class II (T. thermophilus cyt tRNA ${ }^{\text {Tyr }}$, PDB ID: $1 \mathrm{H} 3 \mathrm{E}$ ) and class I tRNAs (yeast cyt tRNA ${ }^{\text {Phe }}$, PDB ID: 1EHZ). The orientation in $B$ is rotated $\sim 90$ degrees with respect to the orientation in $A$. The crystal structures are shown as ribbons passing through the phosphate groups. (Red) AA stem, (yellow) T arm, (green) D arm, (blue) Ac arm. Only atoms of nucleotides involved in relevant interactions are shown (blue). (Large sphere) The phosphate atoms of A14, (small sphere) phosphate atoms of U8. The tertiary interaction A14-U8 closes the loop internal to the elbow between the two secondary structure elements (AA stem-T arm and D arm-AC arm) that make up the tRNA 3-D structure, thus contributing to stabilizing the whole tRNA fold. $(C, D)$ A14-U8 Reverse-Hoogsten base pair in the Methanococcus jannaschii tRNA $^{\text {Tyr }}$ structure (PDB ID: 1J1U) (C) and G14-U8 interaction (present in mutant), modeled from this structure $(D)$ before and after energy minimization. (Green) carbon, (blue) nitrogen, (red) oxygen, (gray) hydrogen. Energetically favorable (dark colors) and unfavorable (light colors) interactions between the two nucleotides occurring before and after minimization are shown: (dark and light blue) hydrogen bonds, (dark red and orange) repulsive interactions. Distances in Angstroms are also shown. Of the two hydrogen bonds present between A14 and U8 (C), only one can be formed by G14 and U8 $(D)$. The oxygen and nitrogen atoms involved in the analyzed interactions are shown with the corresponding positions.

guanine and starting from the same geometry observed for the A14-U8 pair.

The A14-U8 RH geometry is well preserved upon energy minimization. On the contrary, an RH geometry is not expected to be stable for the G14-U8 base pair, because of the proximity of the two carbonyl oxygens in position 2 of U8 and in position 6 of G14, giving repulsive electrostatic interactions. These positions are indicated as $\mathrm{U} 8(\mathrm{O} 2)$ and G14(O6); the same notation is used for the nitrogen positions (see below). Indeed, the results of QM calculations showed that in the presence of G14 a different geometry is obtained, where the G14(N7)-U8(N3) hydrogen bond is still present, although weakened with respect to the A14(N7)-U8(N3) hydrogen bond (the distance increases from $1.56 \AA$ to $1.99 \AA)$, while the two carbonyl oxygens $\mathrm{U} 8(\mathrm{O} 2)$ and $\mathrm{G} 14(\mathrm{O} 6)$ repel to a distance of $4.17 \AA$ (Fig. 4C,D). As a result, the optimized geometry for the G14-U8 base pair is calculated to be $5.5 \mathrm{kcal} / \mathrm{mol}$ less stable than the optimized geometry for the A14-U8 base pair.

The A14G substitution is therefore expected to determine a weakening of the interaction with U8, which might destabilize the conformation of the loop connecting the two double helices and, consequently, the relative orientation of the two double helix secondary structure motifs (AA stem-T arm and AC arm-D arm), ultimately leading to structural variations affecting the whole tRNA fold. Moreover, the G14-U8 optimized geometry is not isosteric with the A14-U8 RH base pair (see Fig. 4), because it shows a significantly different orientation of the glycosidic bonds (Leontis et al. 2002; Lescoute et al. 2005).

At variance with A14, the nucleotide at position 20 interacts with different nucleotides at various positions in different tRNA structures, and it is not involved in conserved tertiary interactions. As an example, in class I yeast tRNA ${ }^{\text {Phe }}$ (PDB ID: 1EHZ), G20(N4) points toward the outside of the molecule and forms a hydrogen bond with the ribose $\mathrm{O} 2$ atom of $\mathrm{A} 21$ (Fig. 5), and in class II Thermus thermophilus tRNA ${ }^{\text {Tyr }}$ (PDB ID: 1H3E), C20(N4) is hydrogen bonded to the phosphate oxygen of G19.

Sequence analyses show that the "invariant" A14 and U8 nucleotides, present in the wild-type human and yeast $\mathrm{mt}$ tRNA $A_{\text {UUR }}^{\text {Leu }}$, are completely conserved in all 39 human and 51 yeast cyt tRNAs and are highly conserved in mt tRNAs, being present in 25 of 27 yeast and 19 of 28 human $\mathrm{mt}$ sequences. Conversely, nucleotide G14 introduced in yeast and human mt tRNA $\mathrm{UUR}_{\mathrm{UU}}^{\mathrm{Lu}}$ by the A14G mutation is present

A

B
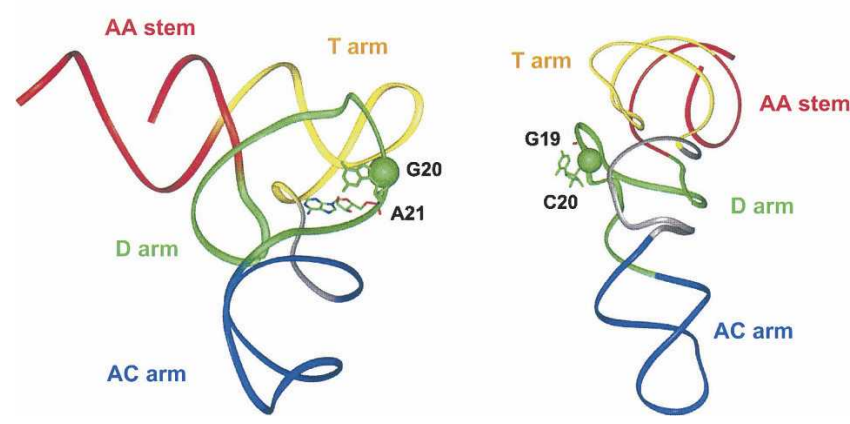

FIGURE 5. Structural effect of the T20C mutation. The nucleotide at position 20 interacts with the ribose moiety of the nucleotide at position 21 in the $3-D$ structure of class I yeast tRNA ${ }^{\text {Phe }}$ (PDB ID: 1EHZ) (A) and with the phosphate backbone of the nucleotide at position 19 in the 3-D structure of class II T. thermophilus tRNA ${ }^{\text {Tyr }}$ (PDB ID: 1H3E) $(B)$. The orientation in $B$ is rotated $\sim 90$ degrees with respect to the orientation in $A$. The crystal structure representation and color coding is as in Fig. 4. Only atoms of nucleotides involved in relevant interactions are shown (green). (Large sphere) The phosphate atom of position 20 . 
only in four human mt tRNAs (tRNA ${ }^{\mathrm{Asn} 1}$, $t R N A^{\mathrm{Gln}}$, tRNA $^{\text {Glu2 }}$, and tRNA $^{\text {Ser1 }}$ ), and the G14-T8 pair is not present in any yeast or human mt or cyt tRNA sequence.

Similarly to A14, T20 is the most frequently occurring nucleotide in yeast and human tRNA sequences. It is conserved in 32 of 39 human and 39 of 51 yeast cyt sequences, in all 27 yeast $\mathrm{mt}$ sequences, and in 7 of 28 human mt sequences; remarkably, however, 17 of 28 human mt tRNAs have a deletion, and not a different nucleotide, at position 20. However, C20, introduced by the T20C mutation in yeast and human mt tRNA $\mathrm{UUR}_{\mathrm{UR}}^{\mathrm{Leu}}$, is not as infrequent as G14: It is present in four yeast and two human cyt sequences and in two human mt sequences.

\section{DISCUSSION}

The aim of the present work is to validate the use of yeast as a model to study the molecular mechanisms by which human base substitutions cause pathologies. We report results on two such substitutions, located at positions 14 and 20 of tRNA $\mathrm{LUU}_{\mathrm{UUR}}^{\mathrm{Leu}}$, respectively, both of which affect the susceptibility of tRNA to aminoacylation as well as tRNA conformation and protein synthesis. The equivalent base substitutions in human tRNAs have been studied by Sohm et al. (2003) and were shown to affect the tRNA acylation in vitro to a different extent. In the present experiments, performed in yeast, the acylation defect is studied in vivo and identified by Northern analysis. This is only possible when the decrease in $\mathrm{mt}$ protein synthesis resulting from the acylation defect is not so severe as to produce a rapid loss of mtDNA. The severity of the defect can be reduced by transferring the mitochondria carrying MELAS-equivalent substitutions, which cause extremely severe respiratory phenotypes, to cells whose nuclear contexts are known to exhibit less mt protein synthesis defect. The reason for the different effect of base substitutions between the studied strains MCC123 and W303-1B is not yet completely understood. We previously showed that some nuclearencoded mt tRNA interactors such as the mt elongation factor EF-Tu and the cognate aminoacyl-tRNA synthetase can suppress even very serious defects due to tRNA base substitutions (Feuermann et al. 2003) and demonstrated, by RT-PCR, that the TUF1 expression levels vary among different yeast strains (De Luca et al. 2006). Based on these results, we hypothesize that the different phenotypes associated with the same mutations in the MCC123 and W303-1B strains might be due to the expression of different amounts of suppressing molecules. In the case of the A14G base substitution, we observed that overexpression of EF-Tu highly increases the retention of mtDNA and allows the detection of the presence of aminoacylated tRNA by Northern analysis (Table 2). However, in these conditions, analysis of the acylation state of tRNA molecules is made very difficult by the almost complete suppression of the defect. By transferring the mutated tRNA to cells with different nuclear contexts, we could determine the acylation state of the mutant A14G, which could not be identified in Northern experiments in the previously studied strain MCC123 due to the rapid and extensive loss of mtDNA, or to the complete suppression of the mutation's effect by overexpression of the TUF1 gene (coding for the elongation factor EF-Tu) or of the NAM2 gene, coding for leucyl-tRNA synthetase. While this enzyme has a catalytic role in the aminoacylation reaction, the elongation factor EF-Tu is not directly involved in this process. The attainment of a complete suppression of the defective phenotype by overexpression of EF-Tu may be due to a "chaperone-like" function of this factor: By interacting with tRNAs whose structures have been altered by the mutations, EF-Tu might help stabilize their nativelike, functional conformations. Indeed, structure analyses, energy calculations, and examination of the frequency of nucleotide occurrence, both at position 14 and at positions involved in conserved interactions with it, indicate that the A14G mutation is likely to induce significant structure alterations in the tRNA $\mathrm{LUR}_{\mathrm{UUR}}^{\mathrm{Lu}}$ (see below).

Our results show that the functionality of $t R N A_{U U R}^{\mathrm{Leu}}$ A14G is significantly lower than that of the T20C mutant. Actually, the A14G mutation produces a very high percentage of rho ${ }^{\circ}$ mutants, resulting in the absence of tRNAs in Northern blot experiments (Feuermann et al. 2003); on the contrary, the T20C mutation, in the same nuclear context (MCC123), produces a lower amount of $\mathrm{rho}^{-} / \mathrm{rho}^{\circ}$ mutants; the delayed and limited growth on glycerol is evidently supported, even at $37^{\circ} \mathrm{C}$, by a very low amount of aminoacylated tRNA $\mathrm{LUR}_{\mathrm{UU}}^{\mathrm{Lu}}$. Conversely, the presence of the aminoacylated form of tRNA $\mathrm{LUR}_{\mathrm{UU}}^{\mathrm{Aeu}} \mathrm{A} 14 \mathrm{G}$ mutant is not sufficient to support $\mathrm{mt}$ protein synthesis in the W3031B nuclear context, whose cells do not grow in glycerol media but exhibit a less severe protein synthesis defect, as shown by the limited production of hho $^{\circ}$ cells (Table 2 ).

These results are in agreement with the results of sequence and structure analyses and energy calculations, according to which $\mathrm{A} 14 \mathrm{G}$ is expected to have a more dramatic effect than the T20C mutation on the structure of tRNA $\mathrm{LuUR}$.

While T20 is not involved in conserved tertiary interactions, A14 participates in a "canonical" tertiary interaction with U8, one of the 15 most conserved tertiary interactions in tRNA molecules. This interaction may have a role in maintaining the tRNA secondary structure and functionality. Puglisi et al. (1993) have shown that the disruption of the A14-U8 base pair in yeast tRNA ${ }^{\text {Asp }}$, due to the A14C substitution, causes a conformational rearrangement of the $\mathrm{D}$ arm, and a dramatic decrease of the tRNA aminoacylation efficiency. Our QM calculations show that the A14G substitution significantly lowers the energy of the 14-8 interaction and changes its geometry. Indeed, the QM optimized G14-U8 base pair is $5.5 \mathrm{kcal} /$ mol less stable than the A14-U8 RH base pair. Due to the 
putative role of the A14-U8 interaction in maintaining the two secondary structure motifs of tRNA molecules (AA stem-T arm and D arm-AC arm) in the correct relative position, the loss of stability and the geometry changes associated to the A14G substitution might result in an overall destabilization and conformation changes affecting the whole tRNA molecule.

Both human and yeast cyt and mt tRNA sequences show a clear preference for $\mathrm{T}$ over $\mathrm{C}$ at position 20 and an even stronger preference for $A$ over $G$ at position 14, possibly due to the stronger structural constraints imposed on A14 than T20. As well as having a role in the maintenance of tRNA structure (both the A14G and T20C mutant show a different electrophoretic migration with respect to wildtype tRNA $\mathrm{LUR}_{\mathrm{UU}}^{\mathrm{Leu}}$ ), the high sequence conservation of A14 and T20 might also be imposed by an involvement of these nucleotides in the recognition of $t R N A_{U U R}^{\text {Leu }}$ by cognate proteins (e.g., aminoacyl-tRNA synthetases, modification enzymes, etc.) and/or the ribosome.

Based on the analysis of known tRNA structures in complex with EF-Tu, neither position 14 nor 20 is expected to be involved in interactions with the elongation factor. Conversely, both A14 and T20 might be identity nucleotides for the cognate leucyl-tRNA synthetase molecule: A14 and U8, involved in a "canonical" interaction with it, are identity nucleotides for $E$. coli $\mathrm{tRNA}_{\mathrm{UUR}}^{\mathrm{Leu}}$; the nucleotide at position 20 is an identity nucleotide for several tRNAs and T20, in particular, for E. coli tRNA $^{\text {Phe }}$ (Giegé et al. 1998); additionally, T3250 has been proposed to be a putative minor identity element for human tRNA $A_{\text {UUR }}^{\mathrm{Leu}}$ (Sohm et al. 2003), and position 20 is within the region recognised by the $\mathrm{mt}$ transcription termination factor (Goto et al. 1992).

In addition to a possible direct effect on recognition by the cognate aminoacyl-synthetase, the acylation defect observed in yeast for the mutated A14G tRNA ${ }_{\mathrm{UUR}}^{\mathrm{Leu}}$ might be mediated by a defective recognition by base-modification enzymes. In humans, the mutation A3243G, equivalent to A14G, together with four other MELAS tRNA $A_{U U R}^{\mathrm{Leu}}$ mutations (G3244A, T3258C, T3271C, and T3291C), has been shown to prevent the modification of U34 to taurine (tm5U34) in the wobble position of the anticodon (Kirino et al. 2004), and the lack of this modification has been proposed to be responsible for the pathological effect. On the other hand, the human mutation equivalent to T20C does not prevent this modification (Kirino et al. 2005). Interestingly, in yeast, wild-type tRNA $\mathrm{LUR}_{\mathrm{UU}}^{\mathrm{Lu}}$ position 34 is also a modified nucleotide (cmnm5). The different behavior of the two mutants might be ascribed to a specific recognition of the A3243 nucleotide, equivalent to A14, which would act as an identity nucleotide for human tRNA modification enzymes and/or to a more dramatic conformational change of the tRNA molecule induced by the A14G with respect to the T20C equivalent mutation.

In conclusion, the present results give important support to the use of the yeast model for the study of pathogenetic base substitutions. Actually, these results show that very severely pathogenetic equivalent base substitutions can be successfully characterized by transferring mutated mitochondria in a nuclear context in which the inhibition of $\mathrm{mt}$ protein synthesis is less severe (which can be determined on the basis of rho $^{\circ}$ production). This is an essential point for the study of such severe mutations. Our results also demonstrate that the molecular mechanism is conserved in human and yeast, at least for the studied mutations. Moreover, the structural and energetic analyses support the results of the Northern experiments and are consistent with the greater reduction of functionality of the A14G with respect to the T20C mutant.

\section{MATERIALS AND METHODS}

\section{Strains, media, and culture conditions}

Saccharomyces cerevisiae strains used in these studies are listed in Table 1. The name of the mutants has the first letter of the corresponding wild-type strain and the location of the specific base substitution. The wild-type strains are: MCC123, W303-1B, and D273-10B/A1. The mitochondrial tRNA $\mathrm{LUR}_{\mathrm{UU}}^{\mathrm{Lu}}$ mutants M/A14G and M/T20C were obtained by cytoductant crosses between the $\mathrm{MCC} 123\left(\mathrm{rho}^{\circ}\right)$ and the biolistic mutants (obtained as described in Feuermann et al. 2003). In these crosses, the mutation kar1-1 prevented nuclear fusion (Conde and Fink 1976). The screening was facilitated by the presence of the ade 2 mutation that enabled us to distinguish colonies with functional mitochondria, which accumulate a red pigment, from colonies with dysfunctional mitochondria, which appeared white (Kim et al. 2002). We crossed the M/mutants with the W303-1B (rho ${ }^{\circ}$ ) strain totally devoid of mtDNA and selected the cells with mutated mitochondria in the W303-1B nuclear context. The same procedure was also performed with other hho $^{\circ}$ strains obtained from rho $^{+}$strains by treatment with ethidium bromide (Deutsch et al. 1974).

Cultures were grown with shaking at $28^{\circ} \mathrm{C}$ in $\mathrm{YP}$ or in minimal medium. YP: $1 \%$ yeast extract (DIFCO), $1 \%$ bacto-peptone (DIFCO) supplemented with $2 \%$ glucose or $3 \%$ glycerol or with $2 \%$ galactose; minimal medium was $0.17 \%$ yeast nitrogen base w/o amino acids (DIFCO), 0.5\% ammonium sulphate, 2\% glucose supplemented with the required auxotrophies at the final concentration of $10 \mathrm{mg} / \mathrm{mL}$. Media were solidified with $1.5 \%$ agar. To measure the production of $\mathrm{rho}^{-} / \mathrm{rho}^{\circ}$ colonies, a large colony grown on glucose plate was inoculated in the same liquid medium. After an overnight growth, the culture was diluted and plated on glucose, and large and small colonies were counted. To discriminate between hho $^{-}$and rho $^{\circ}$ colonies, DAPI staining was performed: In all of the examined cases, colonies with a small size unable to grow on glycerol were found by DAPI staining to be devoid of mt DNA.

\section{DNA and RNA manipulation}

DNA manipulation, restriction enzyme digestion, plasmid engineering, and standard techniques were performed according to Sambrook et al. (1989). 
Mt RNAs were extracted under acidic conditions (sodium acetate buffer $0.3 \mathrm{M}, \mathrm{pH} 5$ ) from purified mitochondria of wildtype and mutant cells grown to mid-log phase in YP media with $2 \%$ galactose at $28^{\circ} \mathrm{C}$ as described by Baldacci and Zennaro (1982). Whenever possible (i.e., when the respiratory defect was not too severe), mitochondria were also isolated after a shift for $4 \mathrm{~h}$ to $37^{\circ} \mathrm{C}$. The alkaline-treated samples were separated in partially denaturing $10 \%$ polyacrylamide- $8 \mathrm{M}$ urea sequencing gels; the electrophoresis conditions and procedures are as described in Francisci et al. (2005) with minor modifications. To improve the separation between acylated and deacylated tRNAs, the gel electrophoresis was run for $48 \mathrm{~h}$. In order to visualize the aminoacyl tRNA $\mathrm{LUR}_{\mathrm{U}}^{\mathrm{Leu}}$ molecule of mutant cells, $20 \mu \mathrm{g}$ of total RNAs extracted from mitochondria were loaded.

These gels, originally reported by Varshney et al. (1991), are the most informative since they permit the determination of whether the mutated tRNA gene is transcribed in vivo and whether the transcript is stable and correctly processed or defective in structure and/or aminoacylation.

\section{Hybridization condition and probes}

The RNA blotting was performed on Amersham's Hybond-XL membranes according to the protocol provided by the supplier. The tRNAs were identified by hybridization with the following oligonucleotides labeled as described by Sambrook et al. (1989): LEU probe (5'-GTAATACATCTTAAGAGTATCG-3'), complementary to residues $26-47$ of the tRNA ${ }^{\text {Leu }}$ gene, and VAL probe (5'-CGTGTGTAAAACGAATGC-3') complementary to residues 24-41 of the tRNA ${ }^{\mathrm{Val}}$ gene. To determine a possible cytoplamic contamination of the mt RNA extracts, the same blots were dehybridized and hybridized with the $5^{\prime}$-end-labeled oligonucleotide (5'-GCATCGTCAATGACTAGTCG-3') specific for the snR39 gene (a small nucleolar RNA very similar in length to tRNA genes).

\section{tRNA sequence and structure analysis}

We analyzed 67 nonredundant human tRNA sequences (39 from cytoplasm and 28 from mitochondria) and 78 nonredundant yeast tRNA sequences ( 51 from cytoplasm and 27 from mitochondria) downloaded from the "Compilation of tRNA sequences and sequences of tRNA genes" (http://www.tRNA.uni-bayreuth.de; Sprinzl and Vassilenko 2005).

Eighty structures of tRNA molecules, containing at least 60 nucleotides (nt), determined by X-ray crystallography at a resolution of $3.3 \AA$ or better, were collected from the PDB data archive (Berman et al. 2000) on May 17th, 2007. Structure analysis and modeling of tRNA mutations were performed using the software InsightII (Dayringer et al. 1986). Yeast tRNA mutations A14G and T20C have been mapped on the structure of: (1) yeast cytosolic tRNA $^{\text {Phe }}$ (PDB ID: 1EHZ, $1.93 \AA$ ), which is considered to be representative of free (not in complex with other molecules) class I cytosolic and prokaryotic tRNAs and of some families of mammalian mt tRNAs, including tRNA $\mathrm{LuU}_{\mathrm{UU}}$; and (2) T. thermophilus cytosolic tRNA ${ }^{\text {Tyr }}$ (PDB ID: 1H3E, resolution $2.9 \AA$ ), as a representative of class II tRNAs. The structure of yeast cytosolic tRNA ${ }^{\text {Phe }}$ was used to extract the starting coordinates for the A14-U8 base pair. The starting coordinates of the G14-U8 base pair were obtained from those of the A14-U8 base pair by manually replacing atoms of the adenine base to convert it into a guanine base. Optimal geometries and accurate interaction energies were then evaluated for both the A14-U8 and G14-U8 base pairs, including the ribose $\mathrm{Cl}^{\prime}$ atoms, by quantum mechanics calculations performed as in Oliva et al. (2006).

\section{ACKNOWLEDGMENTS}

This work was supported by Telethon (GGP030240), by University "La Sapienza" of Rome (C26A039249), by Istituto PasteurFondazione Cenci Bolognetti (grants to L.F. and A.T.), and by GIS Maladies Rares (GISMR0319), by AFM (grants to M.B.-F.).

Received July 24, 2007; accepted October 16, 2007.

\section{REFERENCES}

Baldacci, G. and Zennaro, E. 1982. Mitochondrial transcripts in glucose-repressed cells of Saccharomyces cerevisiae. Eur. J. Biochem. 35: 117-123.

Berlani, E.R., Pentella, C., Macino, G., and Tzagoloff, A. 1980. Assembly of the mitochondrial membrane system: Isolation of mitochondrial transfer ribonucleic acid mutants and characterization of transfer ribonucleic acid genes of Saccharomyces cerevisiae. J. Bacteriol. 141: 1986-1997.

Berman, H.M., Westbrook, J., Feng, Z., Gilliland, G., Bhat, T.N., Weissig, H., Shindyalov, I.N., and Bourne, P.E. 2000. The Protein Data Bank. Nucleic Acids Res. 28: 235-242. doi: 10.1093/nar/ 28.1.235.

Besagni, C. 2007. Étude de mutations dans les ARNmt de la levure Saccharomyces cerevisiae. Application des pathologies humaines. Thèse de Doctorat. In Universitè Paris Sud-Orsay.

Bloomfield, V.A., Crothers, D.M., and Tinoco Jr., I. 2000. Transfer RNA. In Nucleic acids: Structures, properties and functions, pp. 103-110. University Science Books, Sausalito, CA.

Conde, J. and Fink, G.R. 1976. A mutant Saccharomyces cerevisiae defective for nuclear fusion. Proc. Natl. Acad. Sci. 73: 3651-3655.

Dayringer, H.E., Tramontano, A., Sprang, S.R., and Fletterick, R.J. 1986. INSIGHT an interactive molecular graphics package. J. Mol. Graph. 4: 82-87.

De Luca, C., Besagni, C., Frontali, L., Bolotin-Fukuhara, M., and Francisci, S. 2006. Mutations in yeast mt tRNAs: Specific and general suppression by nuclear encoded tRNA interactors. Gene 377: 169-176.

Deutsch, J., Dujon, B., Netter, P., Petrochilo, E., Slonimski, P.P., and Bolotin-Fukuhara, M. 1974. Mitochondrial genetics. VI. The petite mutation in Saccharomyces cerevisiae. Interrelation between the loss of the $\mathrm{rho}^{+}$factor and the loss of drug resistance mitochondrial genetic markers. Genetics 76: 195-219.

Feuermann, M., Francisci, S., Rinaldi, T., De Luca, C., Rohou, H., Frontali, L., and Bolotin Fukuhara, M. 2003. The yeast counterparts of human "MELAS" mutations cause mitochondrial dysfunction that can be rescued by overexpression of the mitochondrial translation factor EF-Tu. EMBO Rep. 4: 53-58.

Francisci, S., De Luca, C., Oliva, R., Morea, V., Tramontano, A., and Frontali, L. 2005. Aminoacylation and conformational properties of yeast mitochondrial tRNA mutants with respiratory deficiency. RNA 11: 914-927.

Giegé, R., Sissler, M., and Forentz, C. 1998. Universal rules and idiosyncratic features in tRNA identity. Nucleic Acids Res. 15: 5017-5035. doi: 10.1093/nar/26.22.5017.

Goto, Y., Tojo, M., Tohyama, J., Horai, S., and Nonaka, I. 1992. A novel point mutation in the mitochondrial tRNA Leu gene in a patient with mitochondrial myopathy, encephalopathy, lactic acidosis, and stroke-like episodes (MELAS). Ann. Neurol. 31: 672-675. 
Kim, G., Sikder, H.A.D., and Singh, K.K. 2002. A colony color method identifies the vulnerability of mitochondria to oxidative damage. Mutagenesis 17: 375-381.

Kirino, Y., Yasukawa, T., Ohta, S., Akira, S., Ishihara, K., Watanabe, K., and Suzuki, T. 2004. Codon-specific translational defect caused by a wobble modification deficiency in mutant tRNA from a human mitochondrial disease. Proc. Natl. Acad. Sci. 101: 15070-15075.

Kirino, Y., Goto, Y., Campos, Y., Arenas, J., and Suzuki, T. 2005. Specific correlation between the wobble modification deficiency in mutant tRNAs and the clinical features of a human mitochondrial disease. Proc. Natl. Acad. Sci. 102: 7127-7132.

Leontis, N.B., Stombaugh, J., and Westhof, E. 2002. The non-WatsonCrick base pairs and their associated isostericity matrices. Nucleic Acids Res. 30: 3497-3531. doi: 10.1093/nar/gkf481.

Lescoute, A., Leontis, N.B., Massire, C., and Westhof, E. 2005. Recurrent structural RNA motifs, isostericity matrices and sequence alignments. Nucleic Acids Res. 33: 2395-2409. doi: 10.1093/nar/gki535.

Mulero, J.J. and Fox, T.D. 1993. Alteration of the Saccharomyces cerevisiae COX2 mRNA 5'-untranslated leader by mitochondrial gene replacement and functional interaction with the translational activator protein PET111. Mol. Biol. Cell 12: 1327-1335.

Myers, A.M., Pape, L.K., and Tzagoloff, A. 1985. Mitochondrial protein synthesis is required for maintenance of intact mitochondria genomes in S. cerevisiae. EMBO J. 4: 2087-2092.
Oliva, R., Cavallo, L., and Tramontano, A. 2006. Accurate energies of hydrogen bonded nucleic acid base pairs and triplets in tRNA tertiary interactions. Nucleic Acids Res. 34: 865-879. doi: 10.1093/ nar/gkj491.

Puglisi, J.D., Pütz, J., Florentz, C., and Giegé, R. 1993. Influence of tRNA tertiary structure and stability on aminoacylation by yeast aspartyl-tRNA synthetase. Nucleic Acids Res. 21: 41-49. doi: 10.1093/nar/21.1.41.

Sambrook, J., Fritsch, E.F., and Maniatis, T. 1989. Molecular cloning: A laboratory manual. Cold Spring Harbor Laboratory Press, Cold Spring Harbor, NY.

Sohm, B., Frugier, M., Brule, H., Olszak, K., Przykorska, A., and Florentz, C. 2003. Towards understanding human mitochondrial leucine aminoacylation identity. J. Mol. Biol. 328: 995-1010.

Sprinzl, M. and Vassilenko, K.S. 2005. Compilation of tRNA sequences and sequences of tRNA genes. Nucleic Acids Res. 33: D139-D140. doi: 10.1093/nar/gki012.

Thomas, B.J. and Rothstein, R. 1989. The genetic control of directrepeat recombination in Saccharomyces: The effect of rad52 and rad1 on mitotic recombination at GAL10, a transcriptionally regulated gene. Genetics 123: 725-738.

Varshney, U., Lee, C.P., and RajBhandary, U.L. 1991. Direct analysis of aminoacylation levels of tRNAs in vivo. Application to studying recognition of Escherichia coli initiator tRNA mutants by glutaminyl-tRNA synthetase. J. Biol. Chem. 266: 24712-24718. 

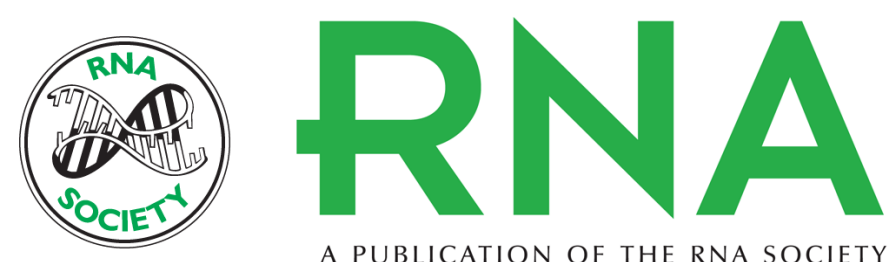

A PUBLICATION OF THE RNA SOCIETY

\section{Yeast as a model of human mitochondrial tRNA base substitutions: Investigation of the molecular basis of respiratory defects}

Arianna Montanari, Céline Besagni, Cristina De Luca, et al.

RNA 2008 14: 275-283

References This article cites 24 articles, 7 of which can be accessed free at: http://rnajournal.cshlp.org/content/14/2/275.full.html\#ref-list-1

Open Access Freely available online through the RNA Open Access option.

License Freely available online through the open access option.

Email Alerting Receive free email alerts when new articles cite this article - sign up in the box at the Service top right corner of the article or click here. 